\title{
SPATIAL AND ENERGY RESOLUTIONS OF A HEXAGONAL ANIMAL PET SCANNER BASED ON LGSO CRYSTAL AND FLAT-PANEL PMT
}

\author{
CHAN MI LEE ${ }^{1,2} \dagger$, SEONG JONG HONG ${ }^{7 *}$, HYUN SUK YOON ${ }^{1,2}$, \\ MIKIKO ITO ${ }^{1,6,8}$, SUN IL KWON ${ }^{1,5}$, SANG KEUN PARK ${ }^{1}$, DONG SOO LEE LE, $^{1,3,6,}$, \\ KWANG SOUK SIM ${ }^{8}$, and JAE SUNG LEE ${ }^{1,2,4,5,6}$ \\ ${ }^{1}$ Department of Nuclear Medicine \\ ${ }^{2}$ Department of Biomedical Sciences \\ ${ }^{3}$ WCU Department of Molecular Medicine \& Biopharmaceutical Sciences \\ ${ }^{4}$ WCU Department of Brain \& Cognitive Sciences \\ ${ }^{5}$ Interdisciplinary Program in Radiation Applied Life Science \\ ${ }^{6}$ Institute of Radiation Medicine, Seoul National University, Seoul, Korea, \\ ${ }^{7}$ Department of Radiological Science, Eulji University, Gyeonggi-do, Korea, \\ ${ }^{8}$ Department of Physics, Korea University, Seoul, Korea \\ ${ }^{\dagger}$ Present address: Korea Institute of Nuclear Safety, Daejeon, Korea \\ *Corresponding author. E-mail : hongseongj@eulji.ac.kr
}

Received October 31, 2010

Accepted for Publication June 01, 2011

The aim of this study was to explore the spatial and energy resolutions of a PET scanner that we have recently developed. The scanner, which consists of six detector modules with 1-layer LGSO crystals, has a hexagonal configuration with a faceto-face distance of $86.4 \mathrm{~mm}$ between two opposite PET modules; such properties facilitate the imaging of small animals. A ${ }^{22} \mathrm{Na}$ point source was employed to estimate horizontal and vertical spatial resolutions. To assess the energy resolution, a uniform ${ }^{18} \mathrm{~F}$ cylindrical phantom was scanned. A software-based spectrum analysis of list-mode data was used to assign a local energy window centered on the photopeak position for every single crystal. For the image reconstruction, an ML-EM algorithm was used. The spatial resolutions at the center of the scanner were $0.99 \mathrm{~mm}$ in the horizontal direction and 1.13 $\mathrm{mm}$ in the vertical direction. The energy resolution averaged over each PMT ranged from 13.3\%-14.3\%, which gave an average value of $13.8 \%$. These results show that this simple system is promising for small animal imaging with excellent spatial and energy resolutions.

KEYWORDS : Positron Emission Tomography (PET), LGSO Crystal, Spatial Resolution, Energy Resolution

\section{INTRODUCTION}

Together with clinical positron emission tomography (PET) scanners designed for human imaging, there is a growing demand for a high-performance PET scanner dedicated to small animals, such as rats and mice. Consequently, many investigators have focused on the development of small animal PET devices with better resolution and sensitivity. Most of the research on animal scanners has focused on achieving the goal of simultaneously improving spatial resolution and sensitivity [1]. To achieve this, several methods to obtain depth of interaction (DOI) information have been proposed [2-6]. In our previous study [7], we demonstrated the feasibility of three-layer depth of interaction (DOI) detectors for a small animal PET using the Monte-Carlo simulation. In this system, three-level DOI encoding was possible due to the relative offset between crystals by one-half a crystal pitch in the $\mathrm{x}$ - and $\mathrm{y}$-directions, along with different decay times.

As an intermediate step toward the full three-layer DOI PET system, we have built a single-layer full-ring system based on an LGSO crystal and a flat-panel PMT. The single-layer PET system has the advantage of simple structure and electronics, with relatively low cost. With a short length and a small cross-sectional dimension of crystals, the single-layer PET system has the potential to achieve $\sim 1 \mathrm{~mm}^{3}$ volumetric resolution without a sophisticated reconstruction algorithm, such as resolution recovery using point spread function and a point source 
[8]. In this paper, we report the results of performance measurement, including those for spatial and energy resolutions. Section 2 describes the single-layer PET system, provides measurements of the spatial and energy resolutions, and outlines a non-uniformity correction algorithm. The results of the energy and spatial resolutions, along with a PMT non-uniformity correction, are described in Section 3. A summary and conclusions are presented in Section 4.

\section{MATERIALS AND METHODS}

\subsection{Single-layer Hexagonal Animal PET}

The PET scanner we have built employs a flat-panel multi-anode PMT (Hamamatsu H9500) and 7-mm long $\mathrm{L}_{1.9} \mathrm{GSO}\left(\mathrm{Lu}_{1.9} \mathrm{G}_{0.1} \mathrm{SiO}_{4}: \mathrm{Ce}\right)$ scintillators with a crosssectional area of $1.5 \times 1.5 \mathrm{~mm}^{2}$, as shown in Fig.1. Fig. 2 shows the hexagonal configuration of the prototype system. One block detector, as shown in Fig. 3, has 841 crystals in a 29 x 29 matrix coupled to a PMT. The gap between crystals was filled with $3 \mathrm{M}$ ESR strips that crossed over each other to form squares. The method, known as the grid method, was first proposed by the Washington group [9]. The flat-panel PMT has 256 anode pixels in a $16 \times 16$ matrix; its effective area is $49 \times 49 \mathrm{~mm}^{2}$. The peak wavelength of the photons generated in the scintillator is $420 \mathrm{~nm}$, which matches the peak wavelength of the PMT [10]. A total of six block detectors were placed to form a hexagonal configuration, and the average face-to-face diameter of the ring was $86.4 \mathrm{~mm}$. Each PMT output signal from the 256 anodes was connected to a charge division circuit, which generated five output signals. Four of the five signals were used to determine the positions of interaction, and one dynode signal served to make a gate signal for coincidence events. A pulse from one dynode channel was switched to a digital pulse with a discrimination threshold of $-50 \mathrm{mV}$ and the pulse width

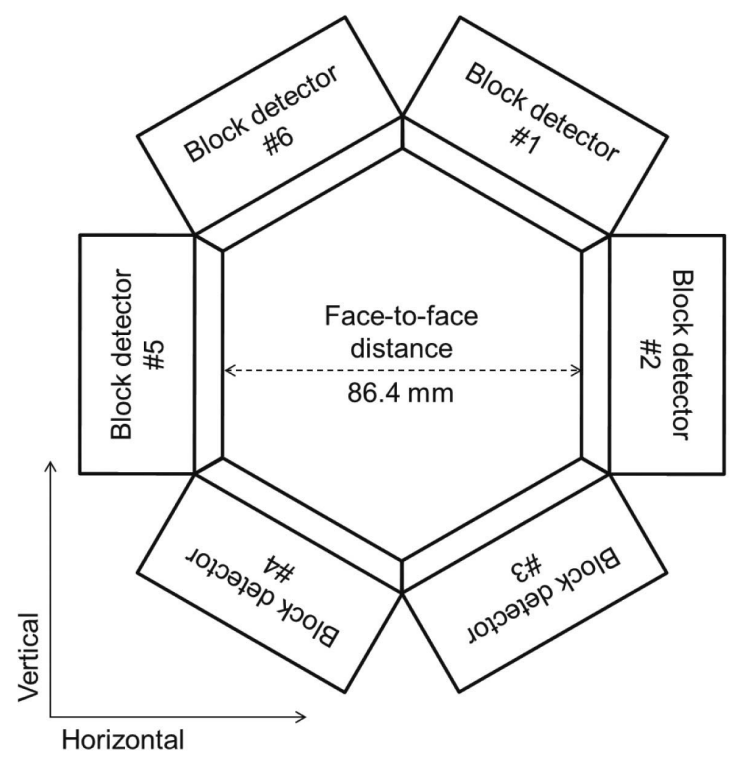

Fig. 2. Schematic Diagram of Prototype Animal PET System.

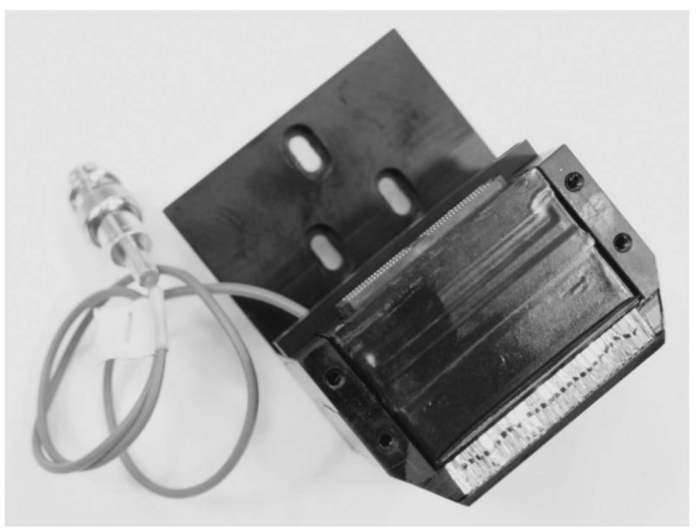

Fig. 3. One of Six PMT-crystal Blocks.
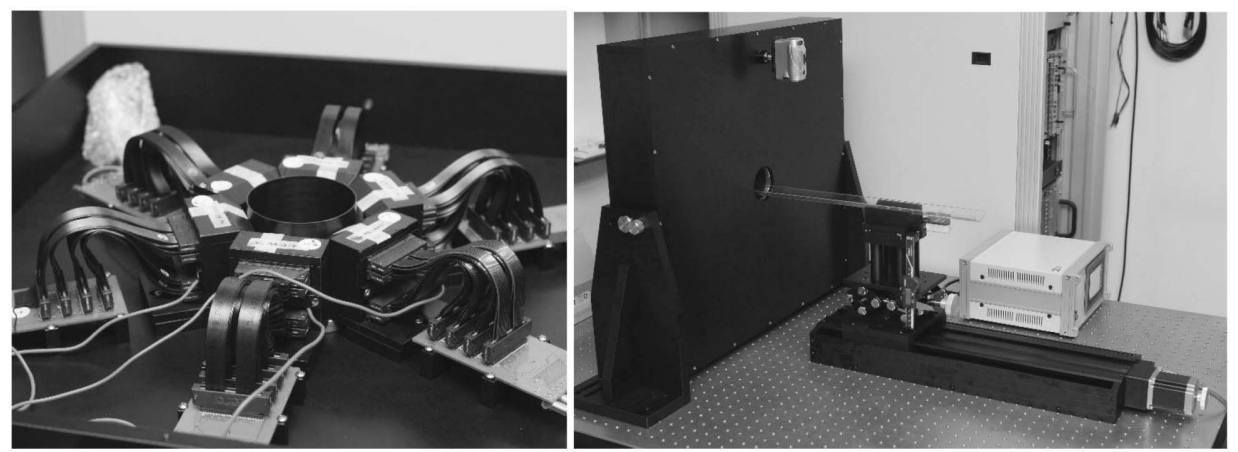

Fig. 1. Prototype Animal PET with Single-layer LGSO Crystal and Flat-panel PMTs. 
of the digital pulse was equal to one-half of the coincidence window. The aim of this conversion was to determine whether or not two of the rectangular pulses from different PMTs would overlap. The digital signal with the duration of the coincidence window was produced at the same time whenever there were overlapping pulses. The coincident logic shown in Fig. 4 was built on a Field Programmable Gate Array (FPGA) using digital signals from six detector modules to generate a GATE signal of $400 \mathrm{~ns}$ to the CAEN VME QDC modules. With the presence of gate signals, the signals containing information about the position of interaction from the charge division circuit were digitized by CAEN QDC modules and digitized data were saved in list-mode. After spectrum analysis and reconstruction utilizing these data, sinograms and reconstructed images can be produced.

\subsection{Detection of Coincidence Events}

The coincidence detector system was implemented on a XILINX Spartan 3 FPGA chip using Verilog HDL. The system had six inputs connected to the discriminated outputs of six PMT blocks, one output signal (gate signal) to indicate coincidence events, and three control signals to set system operation conditions. The coincidence window, gate pulse length, and veto time were set by control signals. The coincidence detector module had a veto function for controlling the data transfer rate of the output signal because the output was connected to the band-limited CAEN VME QDC module.

To implement the coincidence function as digital logic, inputs were converted to pulses having the same length as a coincidence time window. The converted signals were fed into the determiner logic, which consisted of an AND and OR chain, and the coincidence events were detected. The performance of the coincidence detection system depended on the operating clock frequency of the FPGA chip because this process was based on sequential logic, such as that of a register or counter.

The maximum clock frequency of this system was $250 \mathrm{MHz}$; thus, the PET system had a 2 ns minimum coincidence time window when it ran in the double-data rate (DDR) mode. However, we operated the FPGA chip in the $200 \mathrm{MHz}$ single-data rate (SDR) mode, and obtained a 5 ns coincidence time window step. The system also had an approximately $18 \mathrm{~ns}$ output delay due to internal routing delay, thus allowing a controllable coincidence window time and an output pulse length from $5 \mathrm{~ns}$ to $1 \mu \mathrm{s}$ in $5 \mathrm{~ns}$ steps. This also allowed a controllable veto time, which was from $0-25.5 \mu$ s in $0.5 \mu$ s steps.

\subsection{Correction of PMT Non-uniformity}

Since the crystals were placed in slightly different positions in each block detector, and blobs in the flood map of each detector were not evenly spaced, crystal positions from the six detector modules had to be identified by fishnets obtained from a software-based algorithm (Gaussian

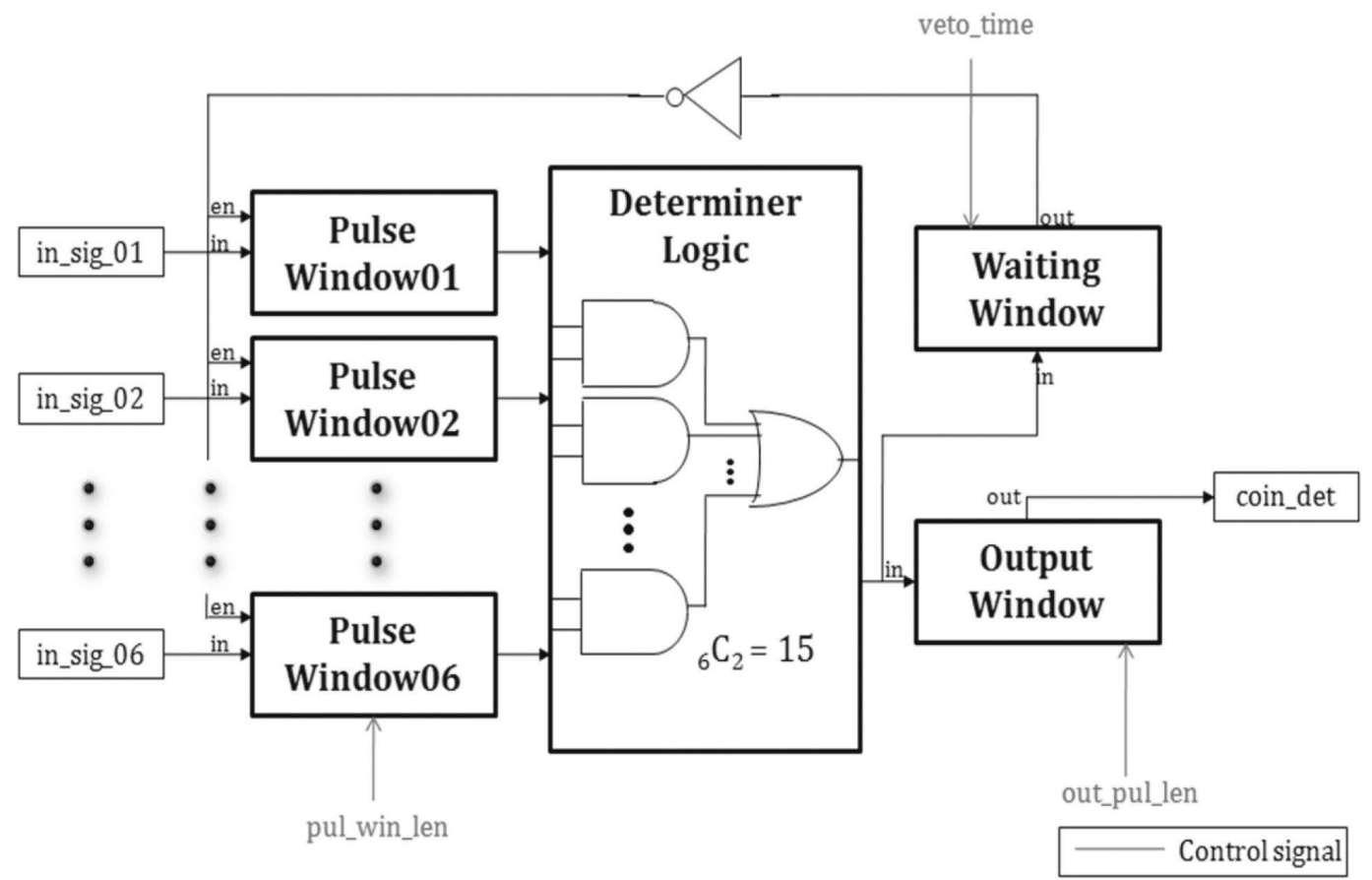

Fig. 4. Generation of Coincident Detection Signal. 
and Laplacian filtering, peak detection, and boundary identification using minimum distance map) [10]. The radioactive decay of ${ }^{176} \mathrm{Lu}$ in the $\mathrm{L}_{1.9} \mathrm{GSO}$ scintillator was used to obtain crystal position maps. Beta-minus particles and associated gamma rays were simultaneously detected by triggering single events from the six detector modules. The crystal maps were used in all analyses, providing the exact positions of the crystals.

In addition, because of the non-uniform efficiency of the sensitive pixels in the multi-anode PMT, the energy spectrum of each individual crystal may have a different photopeak position. When the different spectra of crystals were summed over a PMT, a broad energy distribution was produced. When the broad energy spectrum was used without any correction, the possibility of false discrimination between true and scattered events increased. Therefore, we developed a software package with which a spectrum analysis was performed on list-mode data in order to assign a local energy window centered on the photopeak position for every single crystal determined by the fish-nets. The cylindrical phantom of the ${ }^{18} \mathrm{~F}$ solution scanning data was used to set an energy window for each crystal. Only counts within the local energy window were considered to be valid events. In such a way, variations that were caused by the non-uniform efficiency of PMTs were successfully reduced compared to the case of setting an identical energy window for all sensitive pixels.

\subsection{Spatial Resolution Measured with a Point Source}

The method of evaluating the spatial resolution described here followed the NEMA standard [11]. By imaging a point source in air, we characterized the FWHM of point spread functions (PSFs) from the reconstructed image. Measurement of spatial resolution was performed using a ${ }^{22} \mathrm{Na}$ point source (half-life, $2.6019 \mathrm{y}$ ), with a diameter of $0.25 \mathrm{~mm}$, placed in a plastic disk with a 25$\mathrm{mm}$ diameter (Isotope Products Laboratories). To place the source in a transaxial and axial center, a laser-guided and motor-controlled animal bed was used. The bed position could be controlled technically in units of $10 \mu \mathrm{m}$ in the axial and vertical directions, and $100 \mu \mathrm{m}$ in the horizontal direction. The activity of the source was 81.8 $\mathrm{kBq}$ and data were acquired in list-mode. The list-mode data set were sorted into a 3D sinogram using double sampling without axial compression or sinogram mashing. The data were then rebinned into $2 \mathrm{D}$ data using the single slice rebinning algorithm. For the image reconstruction, the MLEM algorithm was used with a pre-computed system matrix element, which was calculated as the area of intersection between each pixel and the rectangular line of the response (tube of response algorithm). The spatial resolution was measured in a transverse slice at the axial center. The resolutions were specified as a full width at one-half maximum (FWHM), which was estimated using a linear interpolation method [11-12].

\subsection{Energy Resolution Measured with a Cylindrical Phantom}

We assessed the energy resolution using a cylindrical test phantom filled with ${ }^{18} \mathrm{~F}$ solution (half-life, $109.77 \mathrm{~m}$ ). The phantom was made of plastic and had a length of 90 $\mathrm{mm}$ and an inner diameter of $40 \mathrm{~mm}$. The initial activity was $1.85 \mathrm{MBq}$ and the acquisition time was $60 \mathrm{~min}$. The data were acquired in list-mode and the same method described in Section 3 was used for reconstruction.

\section{RESULTS}

\subsection{Correction of PMT Non-uniformity}

Fig. 5 shows a flood map from one of the six detector modules and a projection profile. We obtained an average peak-to-valley ratio of 10.5. A fish net, as shown in Fig. 6,
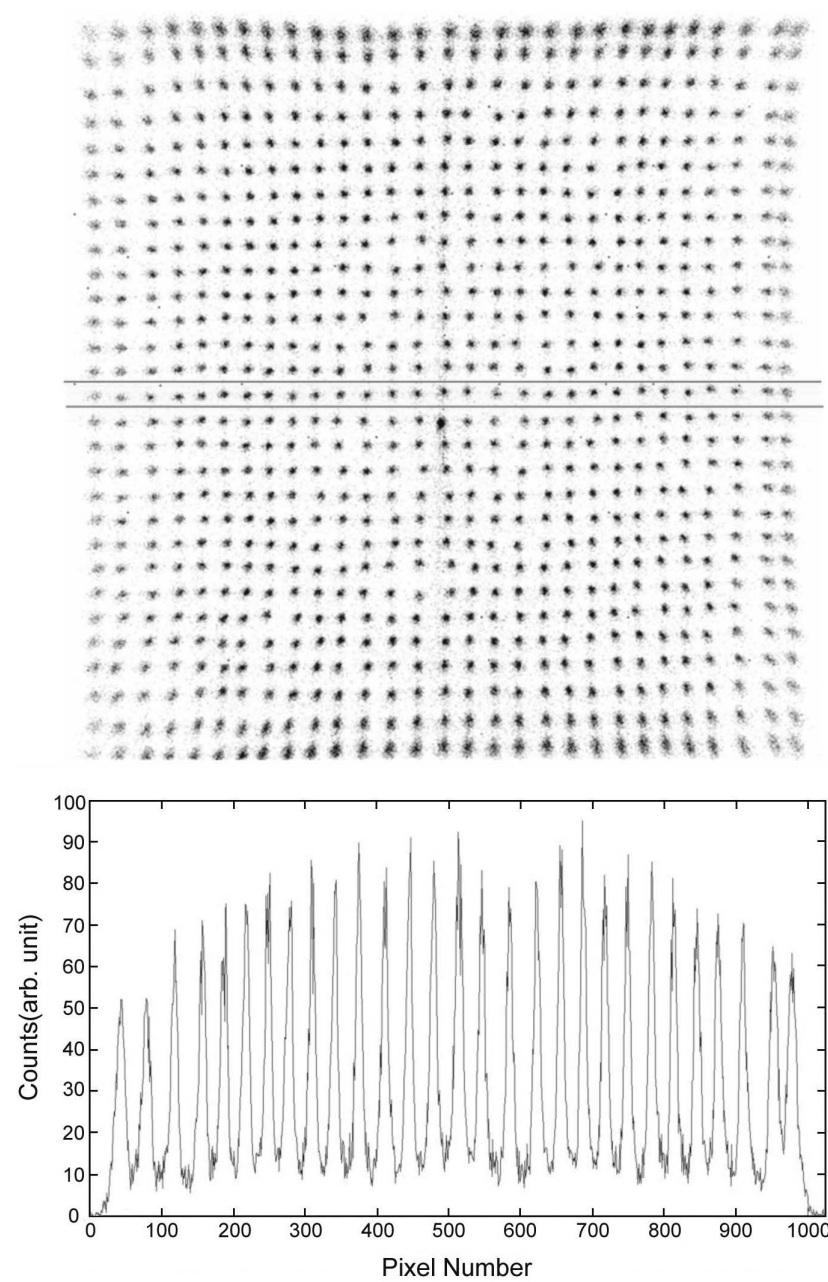

Fig. 5. Crystal Map and Profile. 
was derived from the flood map using the software algorithm [10]. The photopeak position maps from the six block detectors are shown in Fig. 7. Due to the nonuniform light output from the scintillator and the gain difference of the PMT channels, the photopeak position in each crystal was different. Consequently, the integrated energy spectrum of one block detector before photopeak calibration showed a broad photopeak and a significant overlap between the photopeak and Compton scattered events (Fig. 8(a)). After matching the photopeak positions of all individual crystals, the integrated energy spectrum was changed into a more appropriate shape (narrower and higher photopeak and clearer separation of the photopeak events from the Compton scattered events; Fig. 8(b)).

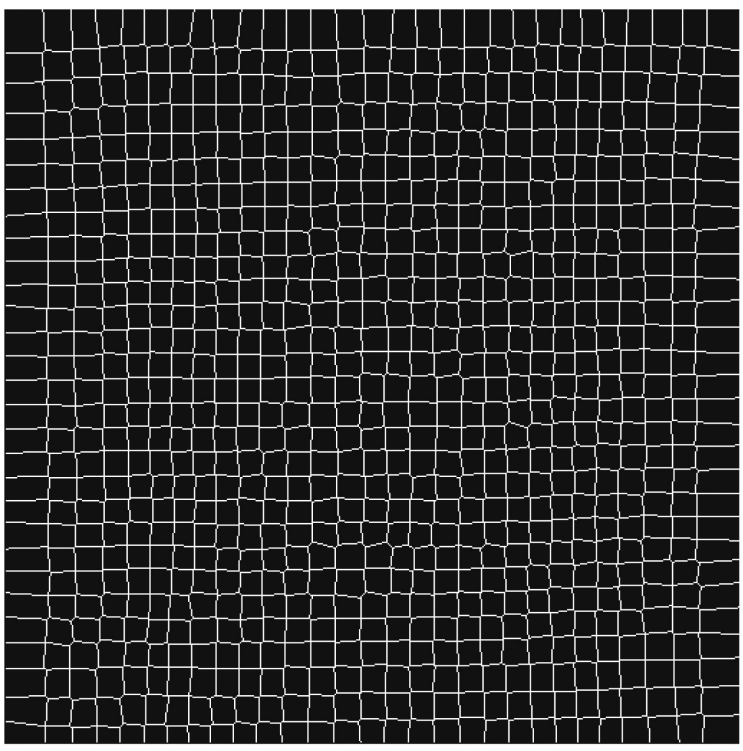

Fig. 6. Fish-net Obtained from the Crystal Map in Fig. 5.
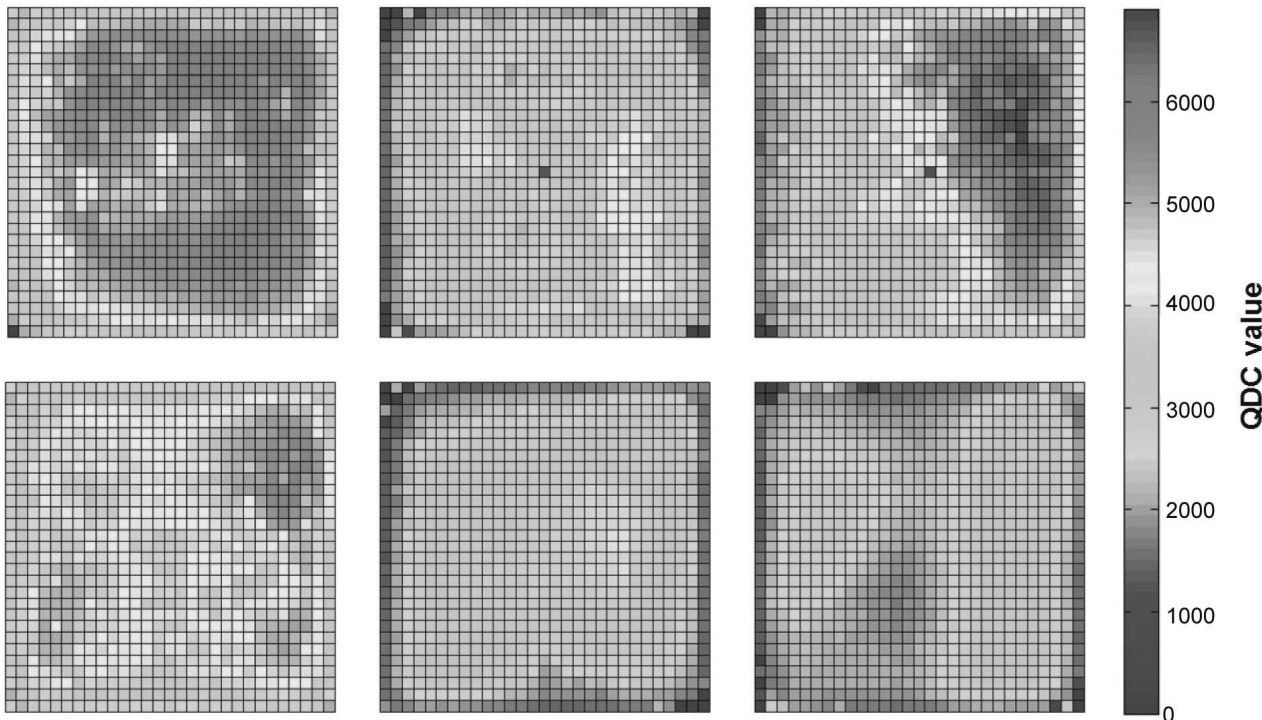

Fig. 7. Photopeak Position Maps from 6 PMT-crystal Blocks. The 1-bit of CAEN VME QDC Corresponds to $25 \mathrm{fC}$. 


\subsection{Spatial Resolution}

We obtained $1.50 \times 10^{6}$ valid coincidence events at the axial and transaxial centers. Fig. 9 shows the sinogram and reconstructed transaxial image; Fig. 10 shows the profiles in the horizontal and vertical directions. The spatial resolutions at the center of the scanner were 0.99 $\mathrm{mm}$ FWHM in the horizontal direction and $1.13 \mathrm{~mm}$ FWHM in the vertical. The FWHM in the vertical direction appears to be worse than the FWHM in the horizontal direction, mainly because of the effect of gaps between

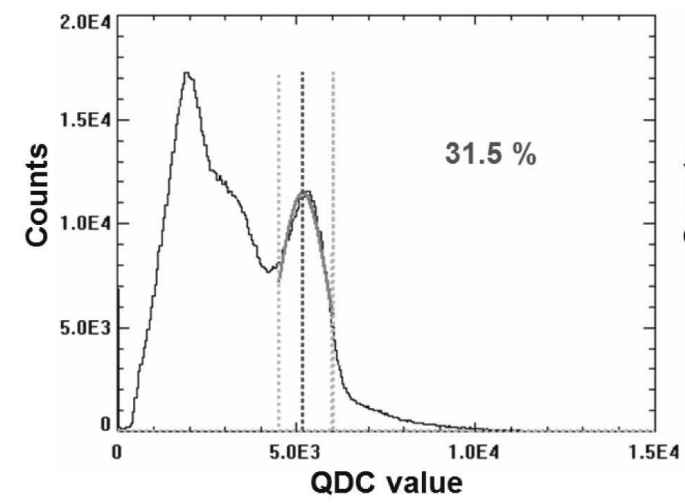

(a)

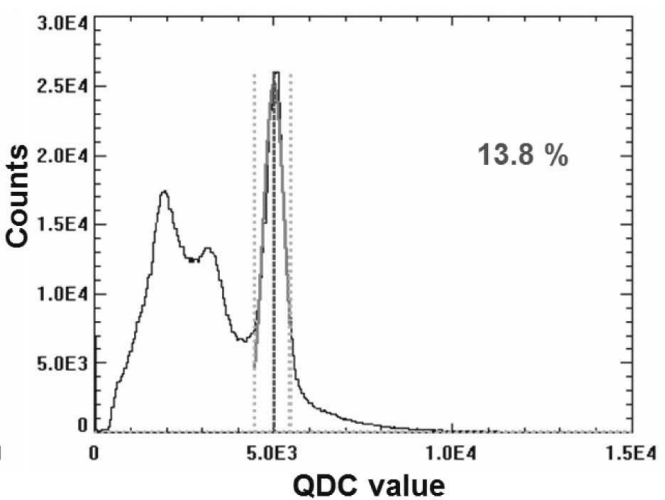

(b)

Fig. 8. Energy Spectrum Over All Crystals in a PMT Before (a) and After (b) Photopeak Calibration.
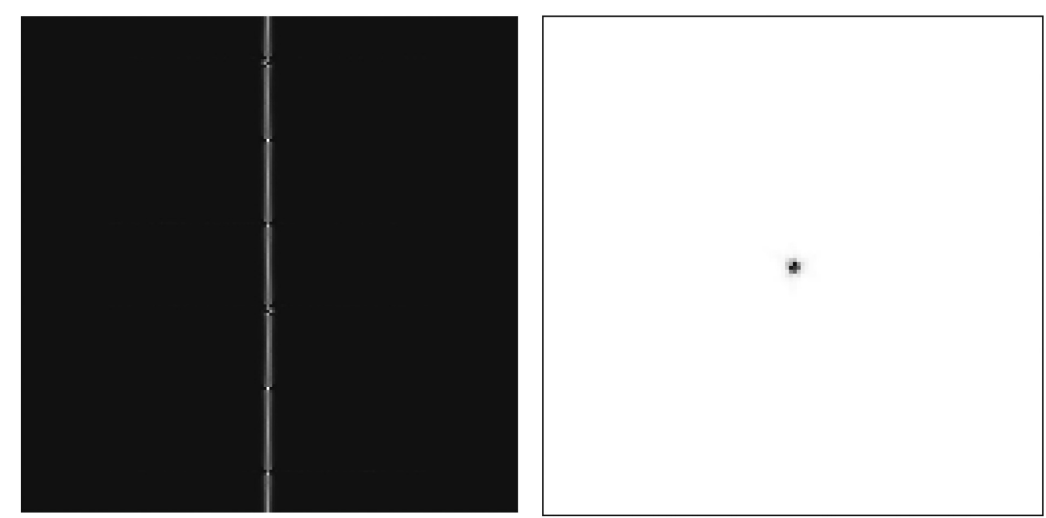

Fig. 9. Sinogram and Reconstructed Image.
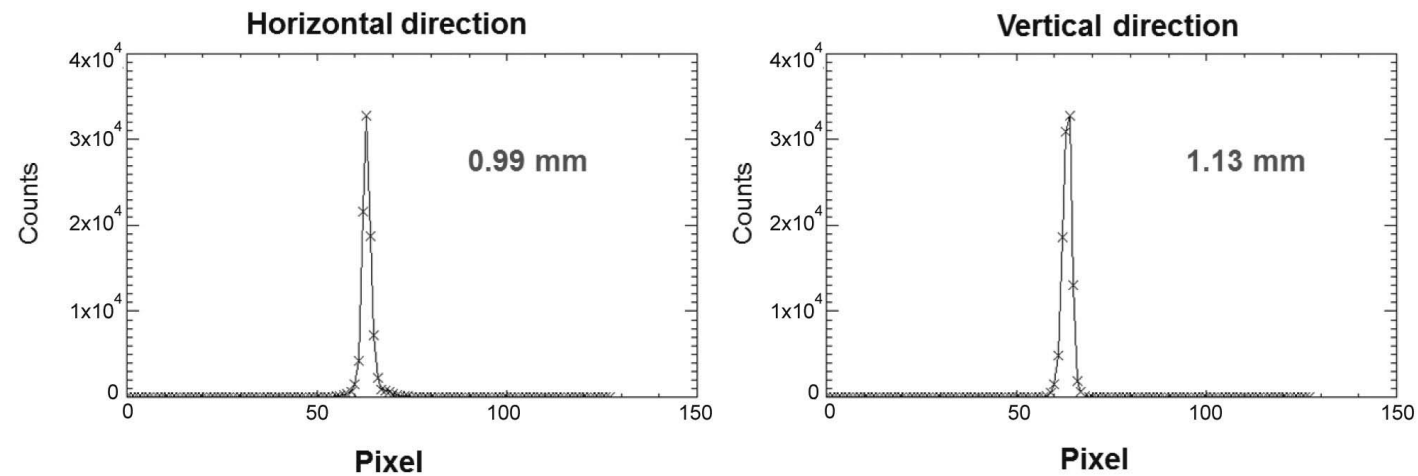

Fig. 10. Profiles of a Point Source to Measure the Spatial Resolution. 
the block detectors. These gaps were unavoidable when we formed the PET system as a hexagonal configuration. Unlike the case for the horizontal direction, in the vertical direction there were spaces between crystal arrays so that annihilation photons escape without interaction. Only adjacent crystals contributed to the detection of photons. The relatively large area of flat-panel PMTs and the requirement of having a smaller face-to-face diameter of the ring for small animal imaging led to significant reconstruction artifacts when a filtered backprojection reconstruction algorithm was applied. Therefore, all scanned PET data were reconstructed using an MLEM reconstruction (iteration number $=32$ ) with exact position information for each LOR element to minimize these artifacts.

\subsection{Energy Resolution}

A total of $1.58 \times 10^{6}$ true coincidence counts were obtained. The distribution of energy resolution for each crystal in all PMTs is presented in Fig. 11. Table 1 describes the mean and standard deviation of energy resolution for all PMTs. The energy resolution averaged over each PMT ranged from 13.3\%-14.3\%, which gave an average value of $13.8 \%$.

\section{SUMMARY AND CONCLUSION}

In this paper, the spatial and energy resolutions of a single-layer hexagonal animal PET system were examined. The system yielded satisfactory results, as follows: an MLEM-based spatial resolution of $0.99 \mathrm{~mm}$ (horizontal) and $1.13 \mathrm{~mm}$ (vertical); and a mean energy resolution of $13.8 \%$. Nevertheless, further studies will have to make great effort for the sake of perfectly completing a threelayer DOI PET. Most importantly, a hardware-based PMT output correction is preferred rather than a softwarebased method. Therefore, correction for non-uniform gain in every channel of the PMT must be taken into account in our next study. We would also like to note that this system should be extended to a three-layer configuration, so as to further improve both resolution and sensitivity.
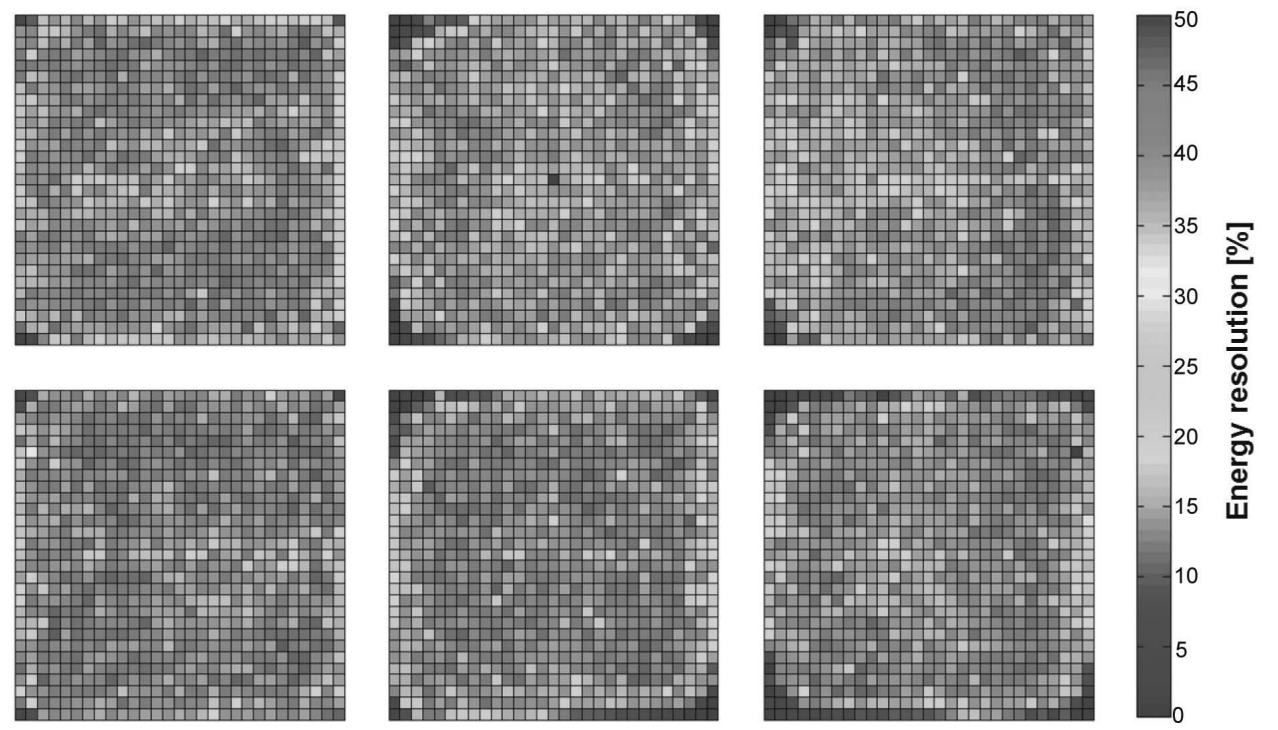

Fig. 11. Distribution of Energy Resolution of Each Crystal.

Table 1. Energy Resolution of 6 PMTs (Mean and Standard Deviation)

\begin{tabular}{c|c|c|c|c|c|c}
\hline Property & 1 & 2 & 3 & 4 & 5 & 6 \\
\hline $\begin{array}{c}\text { Mean energy } \\
\text { resolution [\%] }\end{array}$ & $13.9 \pm 2.32$ & $14.3 \pm 3.32$ & $14.3 \pm 2.54$ & $13.4 \pm 2.10$ & $13.4 \pm 3.07$ & $13.3 \pm 3.21$ \\
\hline
\end{tabular}




\section{ACKNOWLEDGEMENTS}

This work was supported by grants from the Basic Research Program (2006-0049221) and the Atomic Energy R\&D Program (2008-2003852, 2010-0026012) through KOSEF, funded by the Korean Ministry of Education, Science and Technology.

\section{REFERENCES}

[1] D. Rowland, S. Cherry, "Small-Animal Preclinical Nuclear Medicine Instrumentation and Methodology", Semin. Nucl. Med., 38 (2008) 209-222.

[2 ] H. Murayama, H. Ishibashi, H. Uchida, T. Omura, T. Yamashita, "Depth encoding multicrystal detectors for PET", IEEE Trans. Nucl. Sci., 45 (1998) 1152-1157.

[3] M. Streun, G. Brandenburg, H. Larue, H. Saleh, E. Zimmermann, K. Ziemons, H. Halling, "Pulse shape discrimination of LSO and LuYAP scintillators for depth of interaction detection in PET", IEEE Trans. Nucl. Sci., 50 (2003) 344-347.

[4] N. Zhang, C.J. Thompson, "Optimizing position readout circuits in positron emission tomography front-end electronics", IEEE Trans. Nucl. Sci., 50 (2003) 13981403.

[ 5 ] M. Ito, J.S. Lee, S. Il Kwon, G.S. Lee, B. Hong, K.S. Lee, K.S. Sim, S.J. Lee, J.T. Rhee, S.J. Hong, "A Four-Layer DOI Detector With a Relative Offset for Use in an Animal PET System”, IEEE Trans. Nucl. Sci., 57 (2010) 976-981.
[6] M. Ito, J.S. Lee, M.J. Park, K.S. Sim, S.J. Hong, "Design and simulation of a novel method for determining depthof-interaction in a PET scintillation crystal array using a single-ended readout by a multi-anode PMT", Phys. Med. Biol., 55 (2010) 3827-3841.

[7] S.J. Hong, S. Il Kwon, M. Ito, G.S. Lee, K.S. Sim, K.S. Park, J.T. Rhee, J.S. Lee, "Concept verification of threelayer DOI detectors for small animal PET", IEEE Trans. Nucl. Sci., 55 (2008) 912-917.

[ 8 ] V.Y. Panin, F. Kehren, C. Michel, M. Casey, "Fully 3-D PET reconstruction with system matrix derived from point source measurements", IEEE Trans. Med. Imaging, 25 (2006) 907-921.

[9] R.S. Miyaoka, S.G. Kohlmyer, T.K. Lewellen, "Performance characteristics of micro crystal element (MiCE) detectors", IEEE Trans. Nucl. Sci., 48 (2001) 1403-1407.

[10] S. Kwon, S. Hong, M. Ito, H. Yoon, G. Lee, K. Sim, J. Rhee, D. Lee, J. Lee, "Development of Position Encoding Circuit for a Multi-Anode Position Sensitive Photomultiplier Tube", Nuclear Medicine Molecular Imaging, 42 (2008).

[11] NEMA, NEMA Standards Publication NU4-2008: Performace Measurements of Small Animal Positron Emission Tomographs, National Electrical Manufacturers Association, Rosslyn, VA, (2008).

[12] J.S. Kim, J.S. Lee, K.C. Im, S.J. Kim, S.Y. Kim, D.S. Lee, D.H. Moon, "Performance measurement of the microPET focus 120 scanner", Journal of Nuclear Medicine, 48 (2007) 1527-1535. 